\title{
Widespread haploid-biased gene expression enables sperm-level natural selection
}

\author{
Kunal Bhutani1*, Katherine Stansifer'*, Simina Ticau'*, Lazar Bojic', Alexandra-Chloé Villani ${ }^{2,3}$, Joanna Slisz',

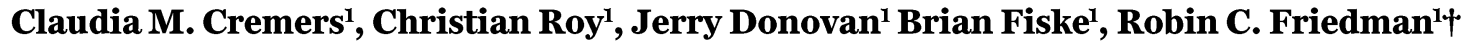 \\ ${ }^{1}$ Ohana Biosciences, Cambridge, MA, USA. ${ }^{2}$ Center for Immunology and Inflammatory Diseases, Center for Cancer Research, Department of Medicine, Massachusetts \\ General Hospital and Harvard Medical School, Boston, MA, USA. ${ }^{3}$ Broad Institute of Massachusetts Institute of Technology and Harvard, Cambridge, MA, USA. \\ *These authors contributed equally to this work.
}

†Corresponding author. E-mail: robin.friedman@ohanabio.com

Sperm are haploid but must be functionally equivalent to distribute alleles equally among progeny. Accordingly, gene products are shared through spermatid cytoplasmic bridges which erase phenotypic differences between individual haploid sperm. Here, we show that a large class of mammalian genes are not completely shared across these bridges. We term these genes "genoinformative markers" (GIMs) and show that a subset can act as selfish genetic elements that spread alleles unevenly through murine, bovine, and human populations. We identify evolutionary pressure to avoid conflict between sperm and somatic function as GIMs are enriched for testis-specific gene expression, paralogs, and isoforms. Therefore, GIMs and sperm-level natural selection may help explain why testis gene expression patterns are an outlier relative to all other tissues.

In diploid organisms, selective pressure acts to maintain equal transmission of alleles to the next generation (1). Mammalian spermatogenesis includes a stage of haploid gene expression, which could lead to functional variation between sperm, resulting in sperm-level natural selection and biased inheritance (24). However, haploid spermatid cells are connected to neighboring cells by cytoplasmic bridges and can share gene products, which can erase phenotypic differences linked to the haploid genome (5) (Fig. 1A). There are few known exceptions to this rule, including a well-characterized mouse selfish genetic element, the $t$ haplotype, which expresses a gene that is not shared across cytoplasmic bridges (6). This drives sperm motility differences and transmission ratio distortion (TRD, i.e., a bias in allelic inheritance) via sperm-level selection.

Given that many cell types have widespread asymmetric mRNA localization $(7,8)$, it is possible that other genes may not freely diffuse through cytoplasmic bridges. This haploid-biased gene expression would be directly observable in allelic expression biases in spermatids. Although most of these biases would be selectively neutral, any affecting sperm function could lead to TRD observable in increased signals of natural selection including rapid fixation of beneficial variants (selective sweeps) and depletion of deleterious alleles.

\footnotetext{
Results

Many mouse genes have allelic expression bias reflecting the haploid genotype in spermatids

We reasoned that if endogenous mRNAs in haploid spermatids are incompletely shared across cytoplasmic bridges, we would observe allelic expression bias correlating to the sperm
}

genotype (Fig. 1A). Since mature sperm are transcriptionally and translationally silent, allelic biases in mature sperm protein correlated with the haploid genotype would correspond to mRNA expression biases at the haploid spermatid stage. We began with an F1 hybrid of distantly related inbred mouse strains, C57BL/ 6 and PWK/PhJ (Fig. 1B). This model has over 20 million fully phased heterozygous SNPs, which aids in accurate and comprehensive allele-specific quantification. We digested testis tissue from four mice to isolate single cells from their cytoplasmic bridges, enriched for haploid cells by flow cytometry, and performed full-length single cell RNA sequencing using a modified SmartSeq2 protocol optimized for sensitive RNA capture. 349 haploid spermatids passed our filters as likely singlets with high-quality quantification, including 110, 95, 77, and 67 from the four mice (fig. S1, A to C). These cells covered the full range of spermatid differentiation up until transcription arrests and the number of genes expressed rapidly decreases (Fig. 1C and fig. S1D).

We compared the expression ratio between the C57BL/6 and $\mathrm{PWK} / \mathrm{PhJ}$ alleles in 12,989 autosomal genes that passed our filters. Visualizing allelic expression in individual haploid cells, we observed biases across tens of megabases of chromosomes, but no consistent bias in diploid cells (Fig. 2A and fig. $\mathrm{S} 2 \mathrm{~A})$. Across all haploid autosomes, there was a correlation of allelic ratios between neighboring genes that gradually decreased with chromosomal distance, and this correlation was completely absent in diploid cells (fig. S1, E and F). We reasoned that this effect could be explained by allele-specific expression correlated with haploid genotype, which we term 
genoinformative expression, combined with DNA recombination degrading this correlation across the chromosome.

Therefore, we developed a Bayesian inference framework (9) to jointly infer the haploid genotypes of each cell and the propensity of each gene to have genoinformative expression (i.e., incomplete sharing across cytoplasmic bridges). Intuitively, this model shares information between genes across an entire chromosome for each cell, meaning even weak and noisy genoinformative expression signals in individual genes can aggregate to yield robust signals across large stretches of a chromosome. Visual inspection confirmed that our inferred genotypes matched the observed expression biases (Fig. 2A and fig. S2A). If the inferred genotypes are accurate, inferred recombination breakpoints should follow the known recombination density of the mouse genome. Indeed, we saw a significant correlation between our inferred recombination density and the published map (10) at a resolution of 10 to 20 megabases $(\mathrm{p}=8 \mathrm{e}-5$, Spearman rank correlation at $10 \mathrm{Mb}$; Fig. $2 \mathrm{~B}$ and fig. S2, B and C).

For individual genes, we observed a wide range of concordance between allelic expression and haploid genotype across cells (Fig. 2C): 8,703 genes, like Sycp3, had less than a 2-fold bias in allelic expression ratio toward the inferred genotype, consistent with sharing across cytoplasmic bridges erasing allelic expression differences; 1,718, such as Smok2b, had more than a 4-fold bias toward their inferred genotype, suggesting minimal sharing across cytoplasmic bridges; and 2,568 had clear but intermediate genoinformativity (2- to 4-fold bias), such as $C c d c 28 a$, suggesting partial sharing through cytoplasmic bridges. We set thresholds for confident genoinformativity at a false discovery rate of $10 \%$ using an empirical background [fig. S2, D and E (9)]. For convenience, we called genes that met these criteria "genoinformative markers" (GIMs), regardless of their effect size.

Of the 12,989 genes for which we could estimate genoinformativity, $41.6 \%$ were confident GIMs and $27.0 \%$ were confident non-GIMs (Fig. 2D, inset). We were unable to make a confident call for the remaining $31.3 \%$ due to marginal signal for genoinformativity. Of the confident genoinformative set, $78.6 \%$ had at least a 2-fold average allelic expression ratio in favor of the allele matching the haploid genotype (Fig. 2D). To summarize genoinformativity, we used a score ranging from 0 to 1 , that reflected the estimated fraction of transcripts originating from a cell's haploid transcription.

\section{GIMs have specific subcellular localization patterns as- sociated with incomplete sharing across cytoplasmic bridges}

We next sought to confirm that strong GIMs corresponded to incomplete sharing across cytoplasmic bridges. The chromatoid body is a membraneless organelle in germ cells that binds to RNA and shuttles across cytoplasmic bridges [Fig. 2E, inset (11)]. Thus, we expected strong GIMs to be depleted from the chromatoid body. Indeed, genes enriched in the chromatoid body (12) had lower genoinformativity scores than other genes (Fig. 2E), and fewer GIMs were enriched in the chromatoid body than expression-matched controls. Although the chromatoid body has not been shown to causally facilitate sharing, this shows that GIMs are preferentially localized elsewhere.

To identify mechanisms for differential localization of GIMs, we compared GIMs to non-GIM controls matched for expression across spermiogenesis. Most mRNA localization is dictated by RNA-binding proteins via 3' UTR binding (13), so we performed an enrichment analysis for motifs of RNAbinding proteins expressed in spermatids (tables S3 and S4) and found 26 motifs significantly enriched in protein-coding GIMs and zero significantly depleted (table S5). Similarly, enrichment analysis of gene ontology and localization annotations (14) identified enrichment for GIMs for specific protein localizations, especially membrane associations and axoneme or other tail localizations (table S6 and S7). Together, these data suggest that RNA-binding proteins bring some mRNAs to specific subcellular locations distal from chromatoid bodies, thus partially avoiding sharing across cytoplasmic bridges in haploid spermatids. Although the majority of GIMs (79\%) were mRNA genes, many other categories of RNA are detected in spermatids and have quantifiable genoinformativity. Among them, RNAs that are retained in the nucleus are expected to exhibit genoinformative expression, for example snRNAs, antisense RNAs, and some lincRNAs. Indeed, these categories had a higher proportion of GIMs than protein-coding genes (Fig. 2F).

As additional validation of our incomplete sharing model for GIMs, we predicted that genes with alleles expressed at different levels (allelic skew e.g., due to expression quantitative trait loci) will have overall expression levels linked to genotype only when they exhibit genoinformativity. Indeed, RNAseq reads that were not allele-specific confirmed this prediction (fig. S2, F to H). We also predicted that GIMs would be expressed higher in haploid round or elongating spermatids than in diploid pachytene cells, and indeed genes peaking in round or elongating stage had higher genoinformativity scores than genes peaking in pachytene or expressed steadily across these stages $(\mathrm{p}<2 \mathrm{e}-4, t$ test; Fig. 2G). Looking at individual cells, GIMs had high concordance with genotype across the entire spermatid pseudotime, so the allelic bias was not limited to a specific stage of haploid development (Fig. 2H).

\section{Sex chromosome genes also exhibit genoinformative ex- pression}

Although our Bayesian method for inferring genotype and genoinformativity only applies to autosomes, we developed a separate method that identified 104 X chromosome genes and $46 \mathrm{Y}$ chromosome genes with genoinformative expression on both the $\mathrm{X}$ and $\mathrm{Y}$ chromsomes after controlling for 
single-cell expression trajectories [fig. S3, A and B (9)]. Results were consistent across mice, and pairwise expression correlations of GIMs were higher on the same chromosome even for GIMs called in other mice (fig. S3, C and D). Cells tended to express higher levels of $\mathrm{X}$ chromosome GIMs and lower levels of $Y$ chromosome GIMs, or vice versa (fig. S3E).

\section{Genoinformativity is conserved between individuals and across mammalian species}

To test if genoinformative expression extends to other mammals, we dissociated testes, isolated haploid spermatids and performed single cell RNAseq (9) using two human tissue donors (302 and 164 cells), two outbred Macaca fascicularis cynomolgus primates (225 and 255 cells), and one Holstein bull (190 cells). Gene expression in spermatids was broadly comparable across all species considered (fig. S4A). Because our method for inferring genotypes relies on sharing information across entire chromosomes, we fully phased each chromosome using a combination of linked-read sequencing and single haploid whole genome sequencing (Fig. 3A and fig. S4B).

We quantified allelic expression of a smaller number of genes for these species than for mice because they had fewer heterozygous sites, ranging from $\sim 13$ million SNPs per cynomolgus individual, to $\sim 3$ million for humans. Nevertheless, we observed comparable quality of our genotype inference, including an overall expression skew in GIMs with allelic expression skew, and false discovery rates under 0.2 for even conservative data shuffles (Fig. 3B and fig. S4, C and D). Genes classified as GIMs in one individual had higher genoinformativity scores in the other individual (Fig. 3B). For each individual, we classified between $31.9 \%$ and $52.3 \%$ of spermatid-expressed genes as confident GIMs (Fig. 3C). The effect sizes were comparable to those seen in mice in each individual, with at least $62.5 \%$ of confident GIMs having at least a 2-fold average expression difference between alleles in favor of the haploid genotype.

Comparing homologs across species with a genoinformativity score in each, we observed significant positive genoinformativity score correlations for every pair of individuals ( $\mathrm{p}<0.03$ for all, Pearson's product-moment correlation test; Fig. 3D). These associations were additive, as genoinformativity scores tended to increase as the number of individuals supporting the GIM call increased (Fig. 3E). This suggests that the features that confer incomplete sharing across cytoplasmic bridges evolve slowly, so that the identities of GIMs tend to be maintained across evolutionary timescales.

\section{GIMs are enriched for signatures of increased natural selection and transmission ratio distortion}

Although it may be a minority, the subset of GIMs that are translated at the haploid stage into proteins that are not completely shared between spermatids could lead to functional differences in sperm and sperm-level selection. This would result in increased evolutionary forces (both purifying and positive selection) acting on GIMs compared to other genes, which would leave detectable signatures in the genome such as selective sweeps in which an allele rapidly spreads in a population. Genes expressed in spermatids already have increased selective forces relative to other genes $(15,16)$, so it is imperative to control for this background. We prepared 20 sets of random mock GIMs from all non-GIM spermatid-expressed genes, or matched for expression patterns across the spermatid pseudotime (fig. S5A). We then cross-referenced a set of selective sweeps in wild mouse populations (17) with GIMs and these non-GIM controls. Compared to the mean and variance across the 20 sets of control genes, GIMs were found in significantly more selective sweep regions than expected by chance ( $p=3 \times 10^{-11}$, z-test) corresponding to an excess of $34 \pm 5.2$ selective sweeps (Fig. 4A, left). Controls should on average be subject to the same selective forces (e.g., sexual selection) but not genoinformativity, suggesting the differences are due to this property.

Because of the small number of GIMs called in humans, we expanded the list of human GIM candidates by inference from cynomolgus. Cynomolgus GIMs were $>6$-fold more likely to be confident GIMs in human than non-GIMs, and there were 1,754 cynomolgus GIMs with no call in human. Across five datasets of human selective sweeps covering different methods timescales (18-22), we find a significant enrichment of GIMs (z-test, $p \leq 0.005$ for each) relative to expression-matched controls, corresponding to over 100 sweeps putatively attributable to genoinformativity (Fig. 4A, right). Examining a broad set of tests for natural selection in the 1000 genomes project data (23), we found significant enrichments in 18/21 tests (Benjamini-Hochberg corrected ztest $\mathrm{p}<0.05$; fig. S5C). Together, this indicates that a subset of GIMs is associated with an increased rate of positive selection over evolutionary time.

Direct observation of sperm-level natural selection in action (transmission ratio distortion, TRD) is difficult because they can reach fixation quickly. However, negative selection in just a few generations can be observed by a depletion of loss-of-function mutations in human populations. We found that genes with the most loss-of-function intolerance (24) were enriched for GIMs relative to controls $(p=1.2 \mathrm{e}-7$, z-test; Fig. 4B). There are also examples in the literature of TRD that are specific to paternal transmission and therefore could be due to sperm-level selection driven by GIMs. Although the mouse strain used does not have the abnormal $t$ haplotype that drives TRD through sperm selection, all three natural Smok family genes quantified were confident mouse GIMs (Smok2a, Smok2b, and Smok3c). Comparing to a review of TRD in mice attributable to gamete selection, four out of nine genes quantified have genoinformativity scores above the 90th percentile [Fig. 4C (25)]. A large study of seven cattle 
breeds that identified sire-specific (paternal) TRD provided a much larger dataset (26). There were 57 GIMs overlapping these paternal TRD loci compared to $22 \pm 3.7$ for expressionmatched controls, for an excess of 35 cases of TRD putatively attributable to GIMs (Fig. 4D). Together, these data provide evidence that a subset of GIMs are associated with increased selective forces on a single-generation level.

Increased testis specificity for a subset of GIMs is consistent with sperm-level natural selection and evolutionary conflict

Sperm-level natural selection poses an evolutionary conundrum: due to its highly specialized function, what is good for the sperm is not necessarily good for the organism. For example, selection for a beneficial allele in sperm may decrease overall fitness if the allele is deleterious in a somatic cell context (fig. S5D). Over evolutionary time, this might make genoinformative expression deleterious for genes with somatic functions, but not for genes uniquely expressed in male reproductive tissue. Supporting this hypothesis, we see that GIMs are more likely to be testis-specific in mouse $\left(p<10^{-22}\right.$, z-test) although not human ( $p=0.47$, z-test; Fig. $4 \mathrm{E}$ ) compared to expression-matched controls. This conflict can also be resolved by separating functions for the gene in germ and somatic cells, for example by gene duplication followed by subfunctionalization (27) (fig. S5D) or testis-specific isoforms. As predicted, GIMs are significantly enriched in paralog families that are predominantly testis-expressed in both mouse $\left(p<10^{-22}\right.$, z-test) and human ( $p=9.6 \times 10^{-22}$, z-test; Fig. $\left.4 \mathrm{~F}\right)$. Human GIMs are also enriched in testis-specific isoforms $(p$ $=0.004$, z-test), and mouse GIMs are significantly more likely to have testis-specific exons ( $p<10^{-22}$, z-test; Fig. $\left.4 \mathrm{G}\right)$.

For GIMs to cause functional differences in sperm implied above, they must incompletely share their proteins across cytoplasmic bridges. In the mouse $t$ haplotype, this occurs partly by translation late in spermiogenesis, so there is less time for the protein to be shared across cytoplasmic bridges (6). We therefore predicted that the testis-specific GIMs which are enriched for causing functional differences in sperm would also be enriched in late translation of their proteins compared to other GIMs. Examining a polysome profiling dataset across mouse spermatogenesis (28), mouse GIMs that were functional candidates based on having testis-specific expression, testis-specific paralogs, or testis-specific exons were enriched for late translation compared to other GIMs $\left(\mathrm{p}=2.6 \times 10^{-43}, \mathrm{p}=8.4 \times 10^{-11}, \mathrm{p}=3.0 \times 10^{-4}\right.$, 2-tailed Fisher's exact test; Fig. 4H). GIMs that overlapped selective sweep regions were not significantly enriched $(p=1,2$-tailed Fisher's exact test). These results suggest that late translation of GIMs is one mechanism by which they may lead to spermlevel functional differences, leading to increased selection and avoidance of evolutionary conflict.

\section{Discussion}

Here, we show that many spermatid-expressed genes are incompletely shared between haploid spermatids, resulting in allelic expression linked to the haploid genotype (genoinformative expression). Our model for the mechanism is that the subcellular localization of RNAs, influenced by RNA-binding protein motifs in the 3 ' UTRs or other mechanisms (8), may result in depletion of GIMs from association with the chromatoid body and cytoplasmic bridges themselves. GIMs are substantially conserved across populations and evolutionary timescales, so we predict that these mechanisms are likely conserved.

In this light, many cases of sperm-level functional differences can be putatively attributed to GIMs $(6,25,29-33)$ Few of these examples have been shown to result in sperm-level natural selection, none are in primates, and it has been widely assumed these are isolated cases and that primate sperm are functionally diploid as a rule. The prevalence of GIMs, representing over a third of spermatid-expressed genes, suggests that additional cases of sperm-level functional heterogeneity associated with genotype will be found, and the evidence for increased selective forces beyond the already elevated background for spermatid-expressed genes suggests that they will have evolutionary consequences in many cases.

While genoinformative expression is common at the RNA level, we do not have direct evidence for how common it is at the protein level and it is probable that many RNA-level GIMs have proteins shared across cytoplasmic bridges. This is consistent with the fact that GIMs that are preferentially translated late in spermiogenesis, when there is less time to be shared across cytoplasmic bridges, are enriched in evidence for avoidance of evolutionary conflict. Even late-translated GIMs may not lead to functional differences in sperm, for example because of delivery of proteins from diploid cells to sperm via epididymal exosomes, potentially masking allelic differences for some genes in mature sperm. Our results are consistent with a small subset of GIMs leading to functional consequences, because although we find significant overlaps with evidence for function, most GIMs do not fall into these categories. Because most GIMs lead to only modest allelic differences (2-4 fold), sperm with these differences may also be functionally equivalent or lead to modest transmission ratio distortion that is challenging to quantify, as is observed for example in mouse Yq deletions or Slx knockdowns $(30,32)$.

Over evolutionary time, the ability of GIMs to lead to sperm-level natural selection and TRD has detectable consequences on the spermatid transcriptome in the avoidance of evolutionary conflict by enrichment for testis-specific expression, testis-specific paralogs, and testis-specific isoforms or exon usage. Genes on sex chromosomes, which are missing from half of haploid spermatids, require some degree of 
sharing for sperm function and specific mechanisms have evolved to facilitate sharing. However, it is not clear that there is sufficient evolutionary pressure to achieve perfect sharing or functional equivalence of spermatids. Because GIMs exhibit stronger positive and negative selective forces, there may even be a benefit from sperm-level selection by optimizing their function faster. In cases of sperm-specific expression, isoforms, or exons, this benefit may not be counterbalanced by a detriment of evolutionary conflict.

The testis-expressed genome has long been a puzzling outlier, including extremely widespread transcription, by far the most tissue-specific gene expression, the most tissue-specific paralogs, and the most rapidly evolving genes (34). A recent article has proposed transcription-coupled repair as an explanation for the widespread transcription feature, particularly for genes with a primary function outside the testis (35). The widespread presence of GIMs complements this idea, raising the possibility that sperm-level selection and resulting evolutionary conflicts are common enough to explain the testisspecific expression features.

\section{Methods Summary}

See supplementary materials for details.

\section{Testes and spermatid isolation}

Mouse testes were all from F1 offspring from a cross of C57BL/6 females and PWK/PhJ males. Adult male cynomolgus primates (Macaca fascicularis), aged 6.3 and 7.2 years old, were used for the non-human primate studies conducted at the University of Kentucky. Both were wild-caught individuals from Mauritius, presumed to be unrelated. For human testes, tissues from deidentified deceased male donors were donated for research, with informed consent from the nextof-kin, through IIAM (the International Institute for the Advancement of Medicine). Bovine testes were obtained from a single adult Holstein individual. Testes were reduced to a single-cell suspension (breaking apart the intracellular bridges between germ cells in the process), using the two-step digestion protocol of (41). Single live spermatids were sorted into 96 -well plates by flow cytometry with in status determined by Hoechst 33342 blue fluorescence.

\section{Single-cell RNA sequencing and QC}

Single cell RNAseq was performed using the Smart-Seq2 protocol with minor modifications described in the supplemental materials. Testes from 4 individual mice were digested, and single cell RNAseq was performed on 144, 140, 96 , and 96 cells per mouse at a mean of 3.3 million reads per cell. 2.8 million reads per cell were mapped and assigned to genes on average. We removed 78 outlier cells by manual inspection, including 42 with fewer than 1 million reads, 26 with fewer than 4,000 genes detected at TPM of 1 (not enough for genoinformativity inference), and 10 with more than 12,000 expressed genes at TPM of 1 (probable doublets). Principal Components Analysis (PCA) and t-Distributed Stochastic Neighbor Embedding (t-SNE) on the remaining cells revealed a mixture of three cell types expressing marker genes for spermatids, spermatocytes, and spermatogonia, respectively (fig. S1, A to C). Spermatocytes were separated from other diploid cells by increased expression of Sycp3. The cluster expressing spermatid markers included $95,110,77$, and 67 cells per mouse, respectively. We used diffusion mapping (42) on these spermatids to define a pseudotime space covering their differentiation process, which corresponded to the first dimension of the diffusion map.

\section{Genoinformativity inference and GIM classification}

In the supplementary material, we describe a Bayesian joint probability model for allele-specific reads given haplotypes in each cell, genoinformativity and allele-specific expression skew of each gene with sequencing counts modeled using a modified beta-binomial distribution. We perform inference of the single-cell haplotypes and genoinformativity levels using numerical optimization.

To classify each gene as a "Confident GIM", "Confident Non-GIM", or "Remaining Gene", we fit the Bayesian model to shuffled data, and compared the posterior distributions for the parameters between real and shuffled data. Cutoffs for confident GIMs were selected based to achieve worst-case false discovery rates of 0.2 for each individual.

\section{Expression-matched control selection}

For all analysis of the properties of GIMs, it is important to control for expression levels in spermatids. The expression trajectory across spermiogenesis was first tabulated for each gene by loess-smoothing the log2 of the TPM expression level (with a pseudocount of 1 and complete dropout considered a zero) against the diffusion map pseudotime value for each cell. All confident non-GIMs expressed in spermatids were considered as controls for all GIMs. Pools of controls were first reduced for each GIM based on two hard filters: first, all genes were equally distributed into 5 bins based on their dropout rates; second, the slope of a linear fit to the expression trajectory was required to differ by no more than 0.2 . For each GIM, 20 mock GIMs having the lowest mean squared difference in log2 expression level were selected from among all non-GIMs remaining in its pool. This resulted in 20 control sets of mock GIMs having similar dropout rates, slope of expression trajectory, and low difference in expression trajectory.

\section{REFERENCES AND NOTES}

1. J. F. Crow, Genes that violate Mendel's rules. Sci. Am. 240, 134-143, 146 (1979). doi:10.1038/scientificamerican0279-134 Medline

2. S. Immler, Sperm competition and sperm cooperation: The potential role of diploid and haploid expression. Reproduction 135, 275-283 (2008). doi:10.1530/REP- 


\section{7-0482 Medline}

3. S. B. Joseph, M. Kirkpatrick, Haploid selection in animals. Trends Ecol. Evol. 19, 592-597 (2004). doi:10.1016/i.tree.2004.08.004

4. F. A. Reed, C. F. Aquadro, Mutation, selection and the future of human evolution. Trends Genet. 22, 479-484 (2006). doi:10.1016/i.tig 2006.07.005 Medline

5. R. E. Braun, R. R. Behringer, J. J. Peschon, R. L. Brinster, R. D. Palmiter, Genetically haploid spermatids are phenotypically diploid. Nature 337, 373-376 (1989). doi:10.1038/337373a0 Medline

6. N. Véron, H. Bauer, A. Y. Weisse, G. Lüder, M. Werber, B. G. Herrmann, Retention of gene products in syncytial spermatids promotes non-Mendelian inheritance as revealed by the t complex responder. Genes Dev. 23, 2705-2710 (2009). doi:10.1101/gad.553009 Medline

7. E. Lécuyer, H. Yoshida, N. Parthasarathy, C. Alm, T. Babak, T. Cerovina, T. R. Hughes, P. Tomancak, H. M. Krause, Global analysis of mRNA localization reveals a prominent role in organizing cellular architecture and function. Cell 131, 174-187 (2007). doi:10.1016/i.cell.2007.08.003 Medline

8. A. R. Buxbaum, G. Haimovich, R. H. Singer, In the right place at the right time: Visualizing and understanding mRNA localization. Nat. Rev. Mol. Cell Biol. 16, 95109 (2015). doi:10.1038/nrm3918 Medline

9. Materials and methods are available as supplementary materials.

10. A. Cox, C. L. Ackert-Bicknell, B. L. Dumont, Y. Ding, J. T. Bell, G. A. Brockmann, J. E. Wergedal, C. Bult, B. Paigen, J. Flint, S. W. Tsaih, G. A. Churchill, K. W. Broman, A new standard genetic map for the laboratory mouse. Genetics 182, 1335-1344 (2009). doi:10.1534/genetics.109.105486 Medline

11. S. Ventelä, J. Toppari, M. Parvinen, Intercellular organelle traffic through cytoplasmic bridges in early spermatids of the rat: Mechanisms of haploid gene product sharing. Mol. Biol. Cell 14, 2768-2780 (2003). doi:10.1091/mbc.e02-100647 Medline

12. O. Meikar, V. V. Vagin, F. Chalmel, K. Sõstar, A. Lardenois, M. Hammell, Y. Jin, M. Da Ros, K. A. Wasik, J. Toppari, G. J. Hannon, N. Kotaja, An atlas of chromatoid body components. RNA 20, 483-495 (2014). doi:10.1261/rna.043729.113 Medline

13. C. Andreassi, A. Riccio, To localize or not to localize: mRNA fate is in $3^{\prime} U T R$ ends. Trends Cell Biol. 19, 465-474 (2009). doi:10.1016/j.tcb.2009.06.001 Medline

14. J. X. Binder, S. Pletscher-Frankild, K. Tsafou, C. Stolte, S. I. O'Donoghue, R. Schneider, L. J. Jensen, COMPARTMENTS: Unification and visualization of protein subcellular localization evidence. Database (Oxford) 2014, bau012 (2014). doi:10.1093/database/bau012 Medline

15. J. M. Good, M. W. Nachman, Rates of protein evolution are positively correlated with developmental timing of expression during mouse spermatogenesis. Mol. Biol. Evol. 22, 1044-1052 (2005). doi:10.1093/molbev/msi087 Medline

16. O. Podlaha, J. Zhang, Positive selection on protein-length in the evolution of a primate sperm ion channel. Proc. Natl. Acad. Sci. U.S.A. 100, 12241-12246 (2003). doi:10.1073/pnas.2033555100 Medline

17. F. Staubach, A. Lorenc, P. W. Messer, K. Tang, D. A. Petrov, D. Tautz, Genome patterns of selection and introgression of haplotypes in natural populations of the house mouse (Mus musculus). PLOS Genet. 8, e1002891 (2012). doi:10.1371/journal.pgen.1002891 Medline

18. A. Refoyo-Martínez, R. R. da Fonseca, K. Halldórsdóttir, E. Árnason, T. Mailund, F. Racimo, Identifying loci under positive selection in complex population histories. Genome Res. 29, 1506-1520 (2019). doi:10.1101/gr.246777.118 Medline

19. D. R. Schrider, A. D. Kern, S/HIC: Robust identification of soft and hard sweeps using machine learning. PLOS Genet. 12, e1005928 (2016). doi:10.1371/journal.pgen.1005928 Medline

20. A. Ferrer-Admetlla, M. Liang, T. Korneliussen, R. Nielsen, On detecting incomplete soft or hard selective sweeps using haplotype structure. Mol. Biol. Evol. 31, 12751291 (2014). doi:10.1093/molbev/msu077 Medline

21. J. Y. Cheng, F. Racimo, R. Nielsen, Ohana: Detecting selection in multiple populations by modelling ancestral admixture components. bioRxiv 546408 [Preprint]. 14 February 2019. https://doi.org/10.1101/546408.

22. K. Munch, K. Nam, M. H. Schierup, T. Mailund, Selective Sweeps across Twenty Millions Years of Primate Evolution. Mol. Biol. Evol. 33, 3065-3074 (2016). doi:10.1093/molbev/msw199 Medline

23. M. Pybus, G. M. Dall'Olio, P. Luisi, M. Uzkudun, A. Carreño-Torres, P. Pavlidis, H. Laayouni, J. Bertranpetit, J. Engelken, 1000 Genomes Selection Browser 1.0: A genome browser dedicated to signatures of natural selection in modern humans. Nucleic Acids Res. 42(D1), D903-D909 (2014). doi:10.1093/nar/gkt1188Medline 24. K. J. Karczewski, L. C. Francioli, G. Tiao, B. B. Cummings, J. Alföldi, O. Wang, R. L. Collins, K. M. Laricchia, A. Ganna, D. P. Birnbaum, L. D. Gauthier, H. Brand, M. Solomonson, N. A. Watts, D. Rhodes, M. Singer-Berk, E. M. England, E. G. Seaby, J. A. Kosmicki, R. K. Walters, K. Tashman, Y. Farjoun, E. Banks, T. Poterba, A. Wang, C. Seed, N. Whiffin, J. X. Chong, K. E. Samocha, E. Pierce-Hoffman, Z. Zappala, A. H. O'Donnell-Luria, E. V. Minikel, B. Weisburd, M. Lek, J. S. Ware, C. Vittal, I. M. Armean, L. Bergelson, K. Cibulskis, K. M. Connolly, M. Covarrubias, S. Donnelly, S. Ferriera, S. Gabriel, J. Gentry, N. Gupta, T. Jeandet, D. Kaplan, C. Llanwarne, R. Munshi, S. Novod, N. Petrillo, D. Roazen, V. Ruano-Rubio, A. Saltzman, M. Schleicher, J. Soto, K. Tibbetts, C. Tolonen, G. Wade, M. E. Talkowski, B. M. Neale, M. J. Daly, D. G. MacArthur; Genome Aggregation Database Consortium, The mutational constraint spectrum quantified from variation in 141,456 humans. Nature 581, 434-443 (2020). doi:10.1038/s41586-020-2308-7 Medline

25. J. H. Nadeau, Do gametes woo? Evidence for their nonrandom union at fertilization. Genetics 207, 369-387 (2017). Medline

26. J. Casellas, J. J. Cañas-Álvarez, A. González-Rodríguez, A. Puig-Oliveras, M. Fina, J. Piedrafita, A. Molina, C. Díaz, J. A. Baró, L. Varona, Bayesian analysis of parentspecific transmission ratio distortion in seven Spanish beef cattle breeds. Anim. Genet. 48, 93-96 (2017). doi:10.1111/age.12509 Medline

27. M. Lynch, A. Force, The probability of duplicate gene preservation by subfunctionalization. Genetics 154, 459-473 (2000). Medline

28. Y. Zhang, C. Tang, T. Yu, R. Zhang, H. Zheng, W. Yan, MicroRNAs control mRNA fate by compartmentalization based on 3' UTR length in male germ cells. Genome Biol. 18, 105 (2017). doi:10.1186/s13059-017-1243-x Medline

29. Y. Zheng, X. Deng, P. A. Martin-DeLeon, Lack of sharing of Spam1 (Ph-20) among mouse spermatids and transmission ratio distortion. Biol. Reprod. 64, 1730-1738 (2001). doi:10.1095/biolreprod64.6.1730 Medline

30. S. J. Conway, S. K. Mahadevaiah, S. M. Darling, B. Capel, A. M. Rattigan, P. S. Burgoyne, Y353/B: A candidate multiple-copy spermiogenesis gene on the mouse Y chromosome. Mamm. Genome 5, 203-210 (1994). doi:10.1007/BF00360546 Medline

31. A. Butler, R. E. Gordon, S. Gatt, E. H. Schuchman, Sperm abnormalities in heterozygous acid sphingomyelinase knockout mice reveal a novel approach for the prevention of genetic diseases. Am. J. Pathol. 170, 2077-2088 (2007). doi:10.2353/aipath.2007.061002 Medline

32. J. Cocquet, P. J. I. Ellis, S. K. Mahadevaiah, N. A. Affara, D. Vaiman, P. S. Burgoyne, A genetic basis for a postmeiotic $X$ versus $Y$ chromosome intragenomic conflict in the mouse. PLOS Genet. 8, e1002900 (2012). doi:10.1371/journal.pgen.1002900 Medline

33. G. Alavioon, C. Hotzy, K. Nakhro, S. Rudolf, D. G. Scofield, S. Zajitschek, A. A. Maklakov, S. Immler, Haploid selection within a single ejaculate increases offspring fitness. Proc. Natl. Acad. Sci. U.S.A. 114, 8053-8058 (2017). doi:10.1073/pnas. 1705601114 Medline

34. K. C. Kleene, Sexual selection, genetic conflict, selfish genes, and the atypical patterns of gene expression in spermatogenic cells. Dev. Biol. 277, 16-26 (2005). doi:10.1016/iydbio.2004.09.031 Medline

35. B. Xia, Y. Yan, M. Baron, F. Wagner, D. Barkley, M. Chiodin, S. Y. Kim, D. L. Keefe J. P. Alukal, J. D. Boeke, I. Yanai, Widespread Transcriptional Scanning in the Testis Modulates Gene Evolution Rates. Cell 180, 248-262.e21 (2020). doi:10.1016/i.cell.2019.12.015 Medline

36. D. Brawand, M. Soumillon, A. Necsulea, P. Julien, G. Csárdi, P. Harrigan, M. Weier, A. Liechti, A. Aximu-Petri, M. Kircher, F. W. Albert, U. Zeller, P. Khaitovich, F. Grützner, S. Bergmann, R. Nielsen, S. Pääbo, H. Kaessmann, The evolution of gene expression levels in mammalian organs. Nature 478, 343-348 (2011). doi:10.1038/nature10532 Medline

37. J. Lonsdale, J. Thomas, M. Salvatore, R. Phillips, E. Lo, S. Shad, R. Hasz, G. Walters, F. Garcia, N. Young, B. Foster, M. Moser, E. Karasik, B. Gillard, K. Ramsey, S. Sullivan, J. Bridge, H. Magazine, J. Syron, J. Fleming, L. Siminoff, H. Traino, M. Mosavel, L. Barker, S. Jewell, D. Rohrer, D. Maxim, D. Filkins, P. Harbach, E. Cortadillo, B. Berghuis, L. Turner, E. Hudson, K. Feenstra, L. Sobin, J. Robb, P. Branton, G. Korzeniewski, C. Shive, D. Tabor, L. Qi, K. Groch, S. Nampally, S. Buia, A. Zimmerman, A. Smith, R. Burges, K. Robinson, K. Valentino, D. Bradbury, M. 
Cosentino, N. Diaz-Mayoral, M. Kennedy, T. Engel, P. Williams, K. Erickson, K. Ardlie, W. Winckler, G. Getz, D. DeLuca, D. MacArthur, M. Kellis, A. Thomson, T. Young, E. Gelfand, M. Donovan, Y. Meng, G. Grant, D. Mash, Y. Marcus, M. Basile, J. Liu, J. Zhu, Z. Tu, N. J. Cox, D. L. Nicolae, E. R. Gamazon, H. K. Im, A. Konkashbaev, J. Pritchard, M. Stevens, T. Flutre, X. Wen, E. T. Dermitzakis, T. Lappalainen, R. Guigo, J. Monlong, M. Sammeth, D. Koller, A. Battle, S. Mostafavi, M. McCarthy, M. Rivas, J. Maller, I. Rusyn, A. Nobel, F. Wright, A. Shabalin, M. Feolo, N. Sharopova, A. Sturcke, J. Paschal, J. M. Anderson, E. L. Wilder, L. K. Derr, E. D. Green, J. P. Struewing, G. Temple, S. Volpi, J. T. Boyer, E. J. Thomson, M. S. Guyer, C. Ng, A. Abdallah, D. Colantuoni, T. R. Insel, S. E. Koester, A. R. Little, P. K. Bender, T. Lehner, Y. Yao, C. C. Compton, J. B. Vaught, S. Sawyer, N. C. Lockhart, J. Demchok, H. F. Moore; GTEx Consortium, The Genotype-Tissue Expression (GTEx) project. Nat. Genet. 45, 580-585 (2013). doi:10.1038/ng.2653 Medline

38. K. Guschanski, M. Warnefors, H. Kaessmann, The evolution of duplicate gene expression in mammalian organs. Genome Res. 27, 1461-1474 (2017). doi:10.1101/gr.215566.116 Medline

39. J. Tapial, K. C. H. Ha, T. Sterne-Weiler, A. Gohr, U. Braunschweig, A. HermosoPulido, M. Quesnel-Vallières, J. Permanyer, R. Sodaei, Y. Marquez, L. Cozzuto, X. Wang, M. Gómez-Velázquez, T. Rayon, M. Manzanares, J. Ponomarenko, B. J. Blencowe, M. Irimia, An atlas of alternative splicing profiles and functional associations reveals new regulatory programs and genes that simultaneously express multiple major isoforms. Genome Res. 27, 1759-1768 (2017). doi:10.1101/gr 220962 117 Medline

40. R. Friedman, K. Bhutani, Code and processed data for: Widespread haploid-biased gene expression enables sperm-level natural selection, Version 1.0), Zenodo.(2020); https://doiorg/10.5281/zenodo.4162383.

41. V. Gaysinskaya, I. Y. Soh, G. W. van der Heijden, A. Bortvin, Optimized flow cytometry isolation of murine spermatocytes. Cytometry A 85, 556-565 (2014). doi: $10.1002 /$ cyto.a.22463 Medline

42. H. Bastos, B. Lassalle, A. Chicheportiche, L. Riou, J. Testart, I. Allemand, P. Fouchet, Flow cytometric characterization of viable meiotic and postmeiotic cells by Hoechst 33342 in mouse spermatogenesis. Cytometry A 65, 40-49 (2005). doi:10.1002/cyto.a.20129 Medline

43. P. Angerer, L. Haghverdi, M. Büttner, F. J. Theis, C. Marr, F. Buettner, destiny: Diffusion maps for large-scale single-cell data in R. Bioinformatics 32, 1241-1243 (2016). doi:10.1093/bioinformatics/btv715 Medline

44. T. M. Keane, L. Goodstadt, P. Danecek, M. A. White, K. Wong, B. Yalcin, A. Heger, A. Agam, G. Slater, M. Goodson, N. A. Furlotte, E. Eskin, C. Nellåker, H. Whitley, J. Cleak, D. Janowitz, P. Hernandez-Pliego, A. Edwards, T. G. Belgard, P. L. Oliver, R. E. McIntyre, A. Bhomra, J. Nicod, X. Gan, W. Yuan, L. van der Weyden, C. A. Steward, S. Bala, J. Stalker, R. Mott, R. Durbin, I. J. Jackson, A. Czechanski, J. A. Guerra-Assunção, L. R. Donahue, L. G. Reinholdt, B. A. Payseur, C. P. Ponting, E. Birney, J. Flint, D. J. Adams, Mouse genomic variation and its effect on phenotypes and gene regulation. Nature 477, 289-294 (2011). doi:10.1038/nature10413 Medline

45. H. Li, R. Durbin, Fast and accurate short read alignment with Burrows-Wheeler transform. Bioinformatics 25, 1754-1760 (2009). doi:10.1093/bioinformatics/btp324 Medline

46. H. Li, B. Handsaker, A. Wysoker, T. Fennell, J. Ruan, N. Homer, G. Marth, G. Abecasis, R. Durbin; 1000 Genome Project Data Processing Subgroup, The Sequence Alignment/Map format and SAMtools. Bioinformatics 25, 2078-2079 (2009). doi:10.1093/bioinformatics/btp352 Medline

47. A. Tarasov, A. J. Vilella, E. Cuppen, I. J. Nijman, P. Prins, Sambamba: Fast processing of NGS alignment formats. Bioinformatics 31, 2032-2034 (2015). doi:10.1093/bioinformatics/btv098 Medline

48. Y. Liao, G. K. Smyth, W. Shi, featureCounts: An efficient general purpose program for assigning sequence reads to genomic features. Bioinformatics 30, 923-930 (2014). doi:10.1093/bioinformatics/btt656 Medline

49. P. Cingolani, A. Platts, L. Wang, M. Coon, T. Nguyen, L. Wang, S. J. Land, X. Lu, D. M. Ruden, A program for annotating and predicting the effects of single nucleotide polymorphisms, SnpEff: SNPs in the genome of Drosophila melanogaster strain w1118; iso-2; iso-3. Fly (Austin) 6, 80-92 (2012). doi:10.4161/fly.19695 Medline

50. J. Salvatier, T. Wiecki, C. Fonnesbeck, Probabilistic programming in Python using PyMC3. PeerJ Comput. Sci. 2, e55 (2016). doi:10.7717/peerj-cs.55

51. E. Y. Liu, A. P. Morgan, E. J. Chesler, W. Wang, G. A. Churchill, F. Pardo-Manuel de
Villena, High-resolution sex-specific linkage maps of the mouse reveal polarized distribution of crossovers in male germline. Genetics 197, 91-106 (2014). doi:10.1534/genetics.114.161653 Medline

\section{ACKNOWLEDGMENTS}

We thank Abhishek Sarkar for helpful comments. Funding: All funding was provided by Ohana Biosciences, Inc. Author contributions: BF, RF, and KS conceived the project. KS, ST, LB, CV, JS, CC, and JD performed the experiments. KB, CR, and $\mathrm{RF}$ analyzed the data. KB created the Bayesian method. RF and KB drafted the manuscript, and KS, LB, and ST contributed to revisions. Competing interests: $\mathrm{KB}, \mathrm{KS}, \mathrm{ST}, \mathrm{LB}, \mathrm{JS}, \mathrm{CC}, \mathrm{CR}, \mathrm{JD}, \mathrm{BF}$, and RF are current or former employees of Ohana Biosciences. CV is a former consultant for Ohana Biosciences. The study was funded entirely by Ohana Biosciences. Ohana Biosciences has filed patent applications (62/806,594 and 63/027,721) based on this work. Some authors are current employees of other companies that have no relationship to this work: KB and CR, Takeda Oncology; ST, Alnylam Pharmaceuticals; LB, enGene; JS, Agenus; BF, Mythic Therapeutics; RF, Dragonfly Therapeutics. Data and materials availability: All non-human RNAseq and DNAseq are available on NCBI's Sequence Read Archive (SRA) under project accession PRJNA667172, and human data are available in dbGAP under accession phs002279.v1.p1. Code for the analysis, including code for reproducing the figures, and processed data, is available on Zenodo (40).

\section{SUPPLEMENTARY MATERIALS}

science. sciencemag.org/cgi/content/full/science.abb1723/DC1

Materials and Methods

Figs. S1 to S5

References 41-51

Tables S1 to S11

MDAR Reproducibility Checklist

6 February 2020; accepted 18 December 2020

Published online 14 January 2021

10.1126/science.abb1723 

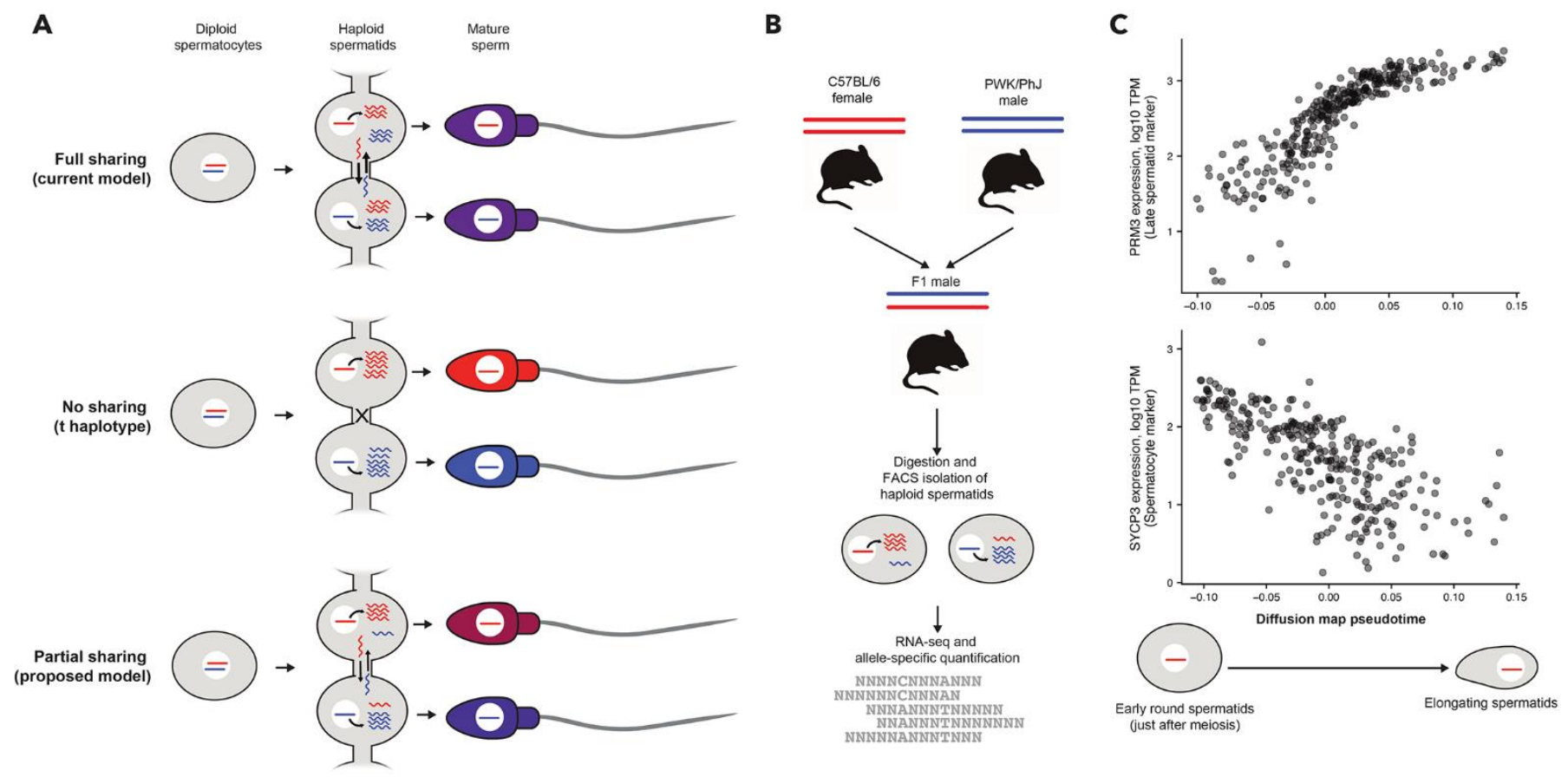

Fig. 1. Single-cell sequencing of haploid spermatids for assessing allelic bias. (A) Models for allelic expression bias informative of the haploid genotype (genoinformative expression). Spermatids are linked by cytoplasmic bridges. DNA is represented as straight lines with color representing an allele, RNA is represented as wavy lines. Sperm color represents the degree of functional links to the allelic genotype. (B) Experimental setup for single cell RNAseq. (C) Pseudotime analysis shows haploid spermatids covered a range from the early round stage (low expression of protamines) to the elongating phase (low expression of SYCP3). 
A

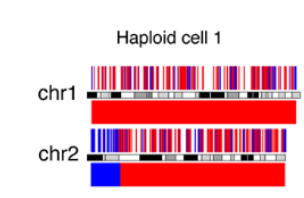

Haploid cell 2

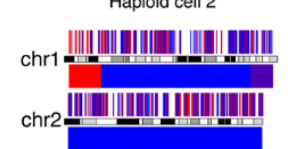

Paternal haplotype

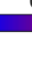

B

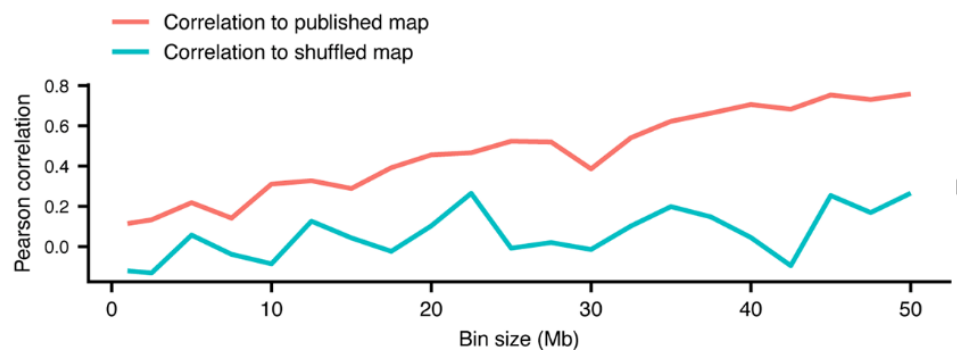

D

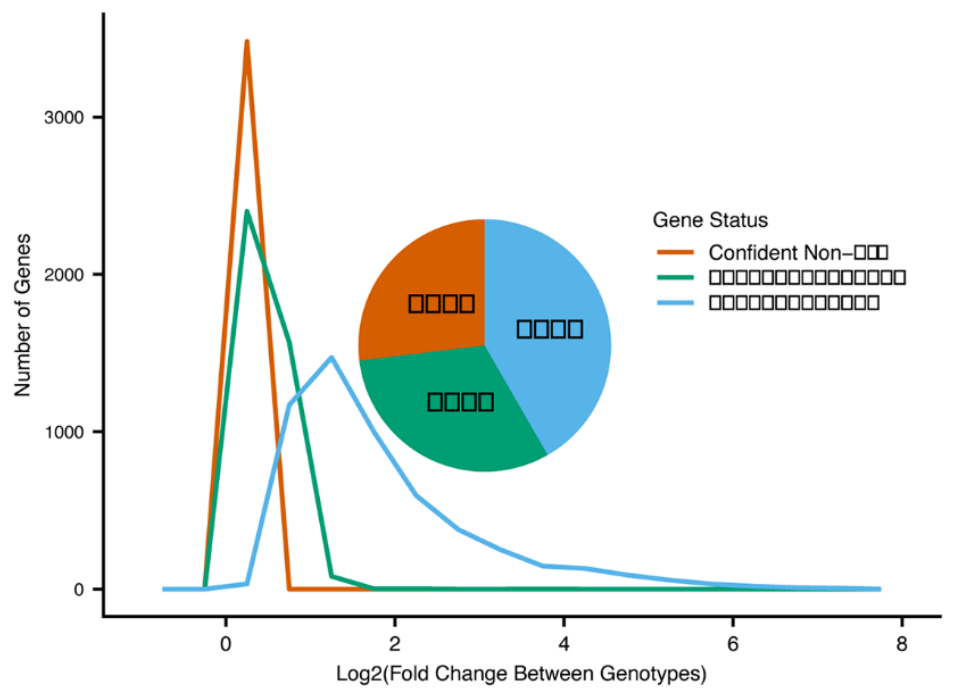

$\mathbf{F}$
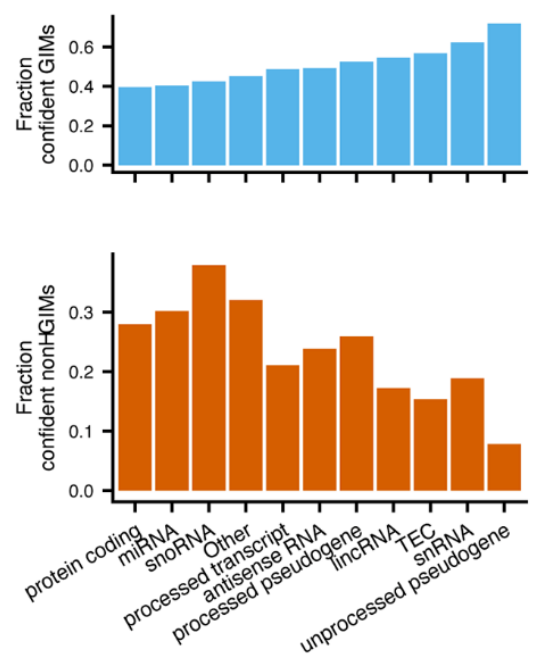

Ensembl Biotype
G

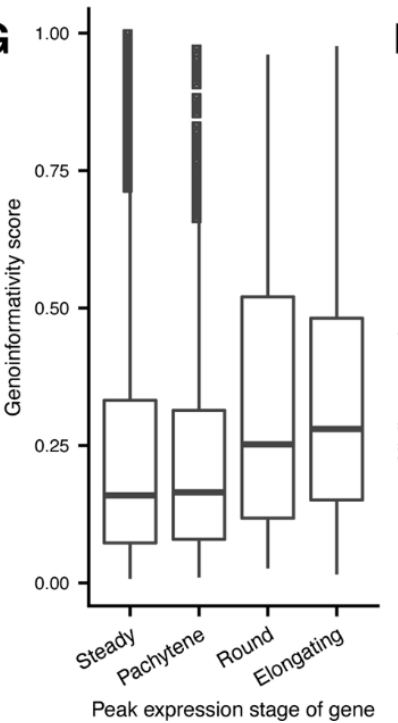
Diploid cell 1
chr1
C

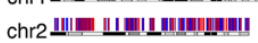

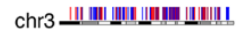

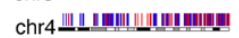

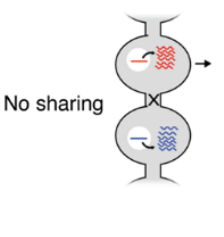

E

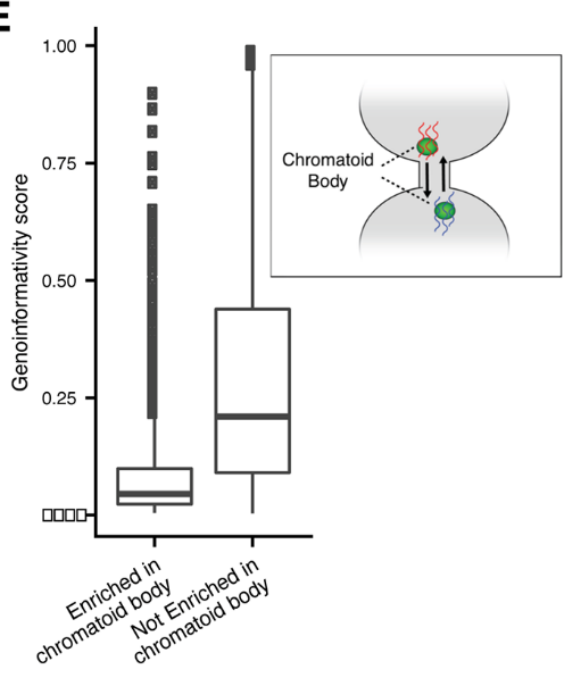

H $\quad$ Confident NonHGIMs

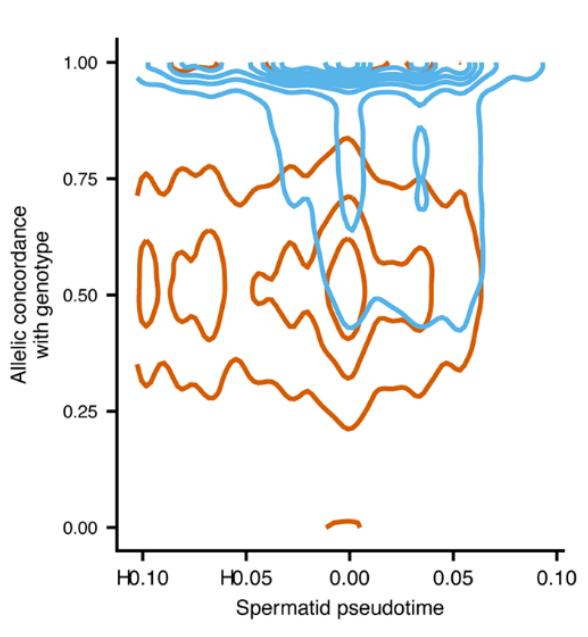


Fig. 2. Mouse genes exhibit genoinformative expression. (A) Visualization of allelic bias in the first two chromosomes of two representative haploid cells. Each expressed gene is represented by vertical line colored by allelic ratio. Below each chromosome is the genotype inferred by our Bayesian method. (B) Correlations between inferred recombination densities and a mouse recombination map (7) or a control with recombination densities shuffled between all bins. (C) Example genes illustrating different levels of genoinformative expression (right) with their models of sharing (left). (D) GIM classification of all genes. Histogram shows the log2 of the expression ratio between the concordant allele (i.e., matching the genotype) over the discordant allele on average across cells. Inset: the total number of genes classified in each category of genoinformative expression. (E) Genoinformativity scores, representing the estimated fraction of transcripts originating from a cell's haploid transcription, for genes enriched in the chromatoid body or not. Inset: depiction of the chromatoid body's proposed role in shuttling mRNAs across cytoplasmic bridges in haploid spermatids. (F) Fraction of genes classified as GIMs or non-GIMs by type. (G) Genoinformativity score distributions for genes by peak expression during late spermatogenesis. $(\mathrm{H})$ Contour plot for genoinformativity (measured by concordance between RNA and genotype) across the spermatid pseudotime for GIMs and non-GIMs. 
A
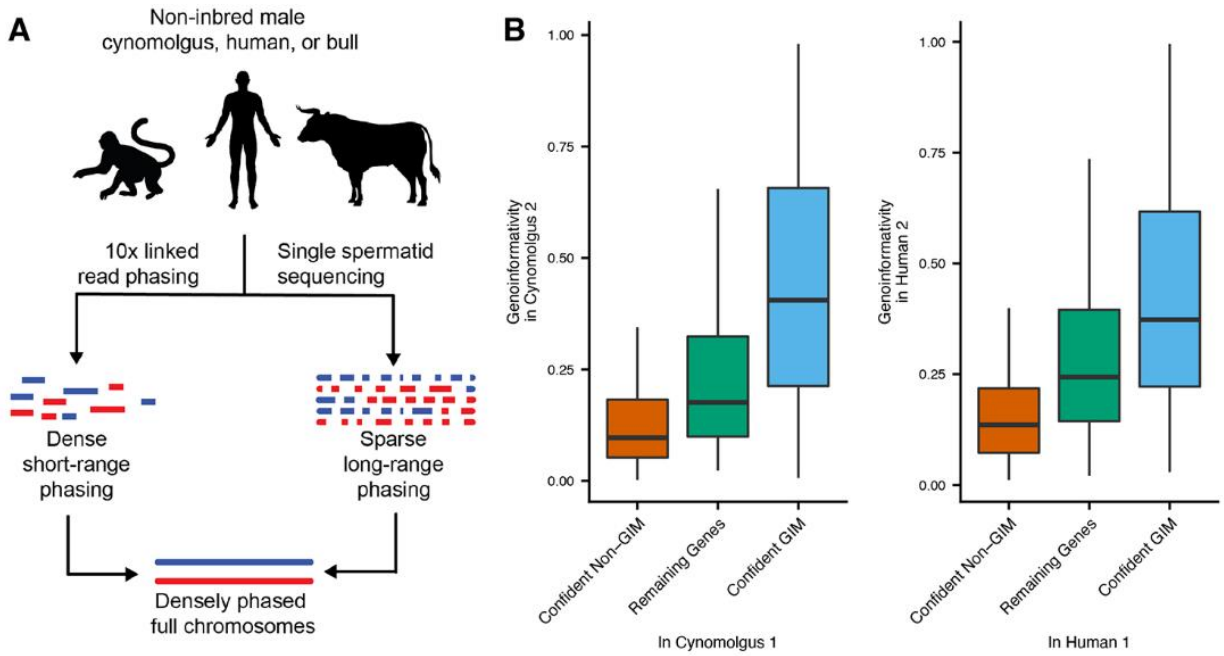

C

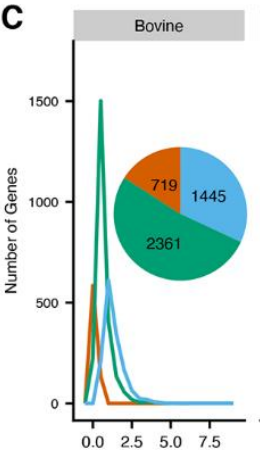

Cynomolgus 1

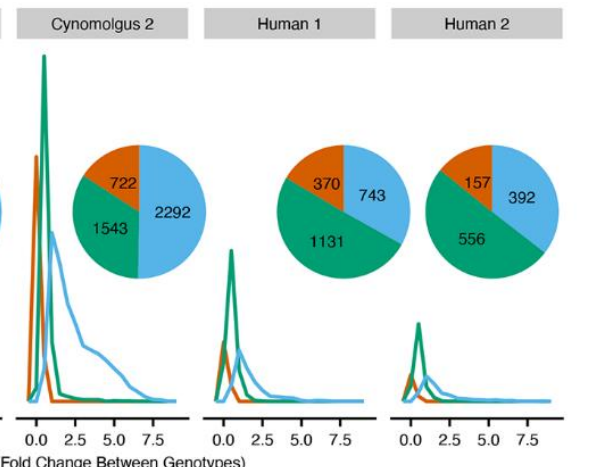

Gene Status

- Confident Non-GIM

- Remaining Genes

- Confident GIM

D

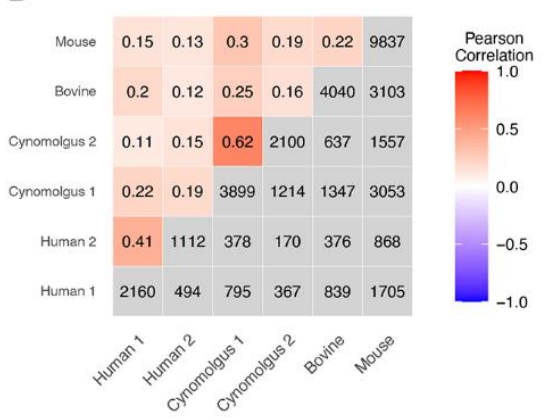

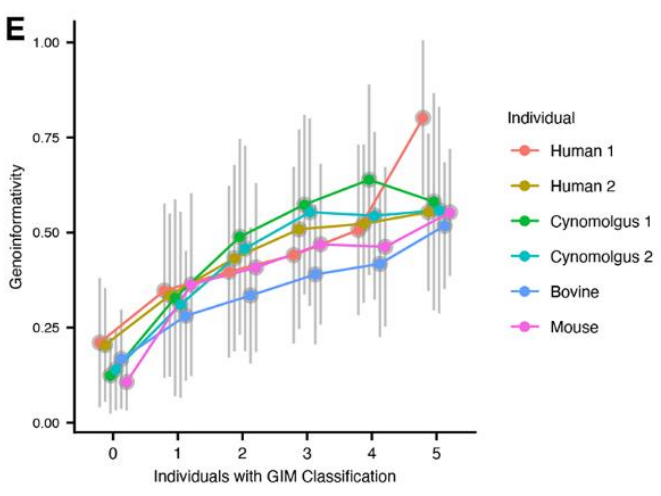

Fig. 3. GIMs are conserved between individuals and across species. (A) Overview of strategy for full-length chromosome phasing. (B) Genoinformativity scores are higher in Cynomolgus 2 for Cynomolgus 1 GIMs than non-GIMs (left, $p<2.2 \times 10^{-16}, t$ test), or in Human 2 for GIMs vs. non-GIMs called in Human 1 (right, $p<2.2 \times 10^{-16}, t$ test). (C) Genoinformative expression classification of all genes as in Fig. 2D, for each individual sequenced. Histogram shows the log2 of the expression ratio between the concordant allele and the discordant allele on average, where the concordant allele matches the inferred genotype. Inset: the total number of genes classified in each category of genoinformative expression. (D) Upper-triangle: correlations between genoinformativity scores of homologs across each individual $(p<0.03$ for all, Pearson's product-moment test). Lower-triangle: number of homologous genes with genoinformativity calls used for pairwise correlation calculation. (E) Mean genoinformativity scores \pm standard deviation in each individual, stratified by the number of individuals calling it a GIM. 

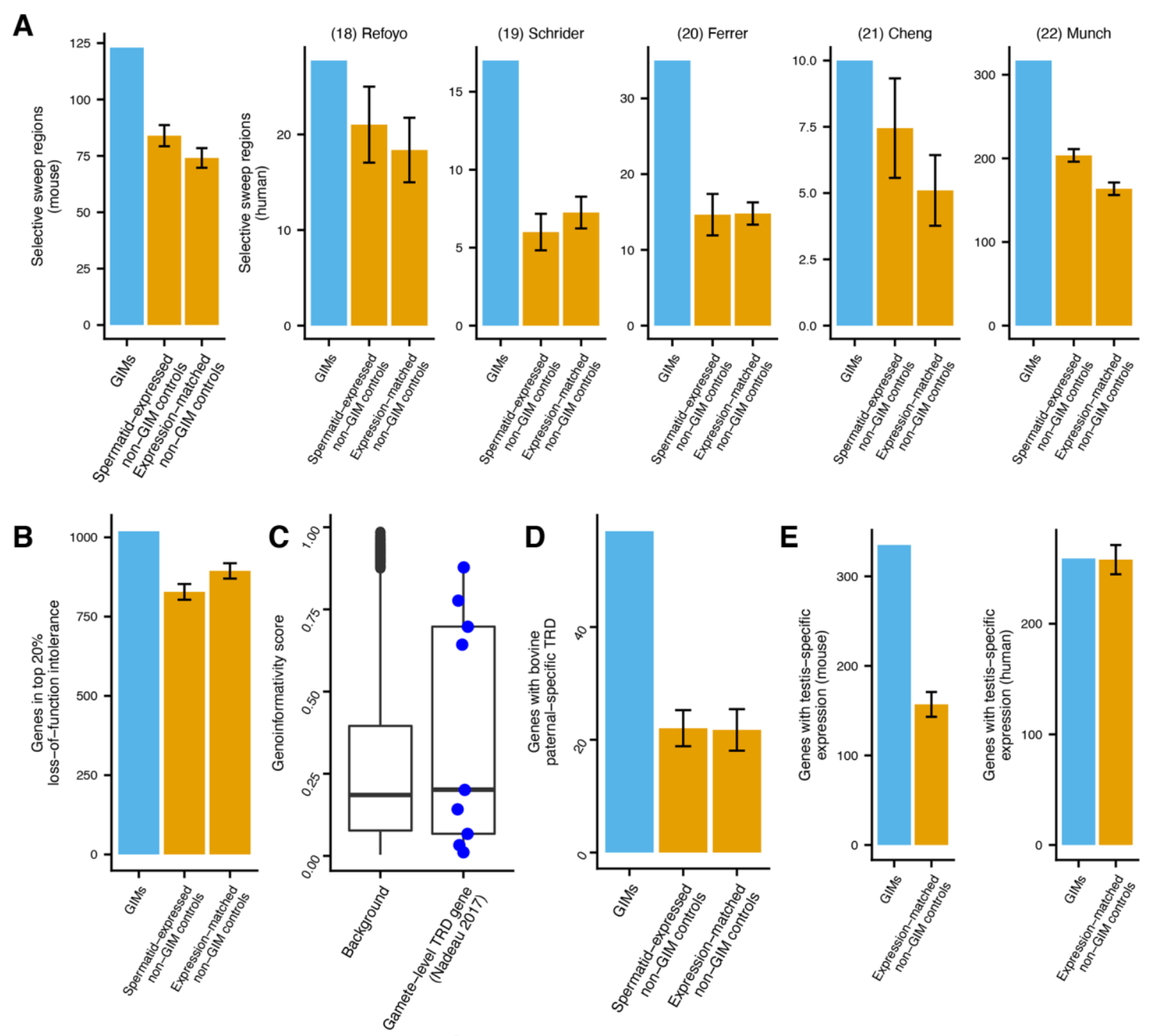

$\mathbf{F}$
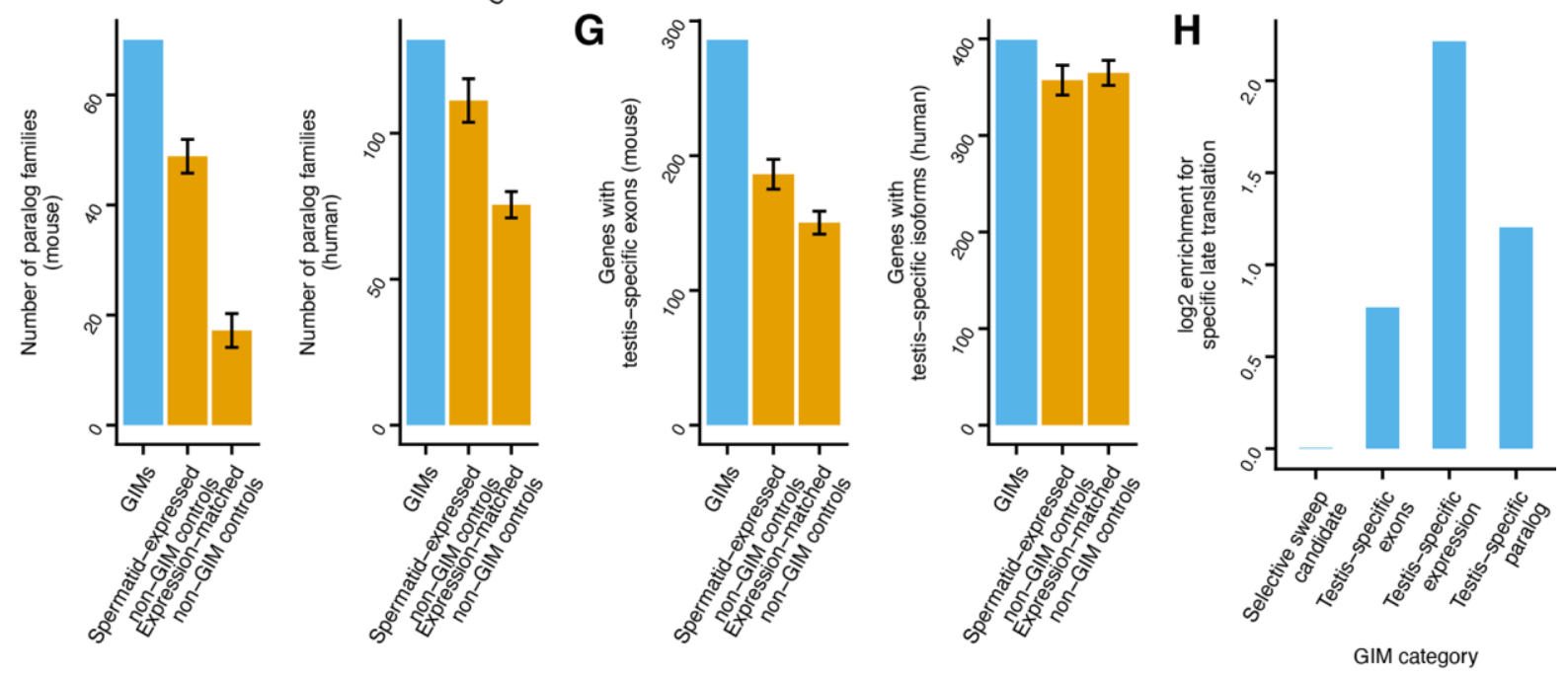
Fig. 4. GIMs are associated with sperm-level natural selection and evolutionary conflict. Human GIMs in this figure are a mixture of directly-measured GIMs and those inferred from Cynomolgus. (A) GIMs are enriched in selective sweep regions in mouse (17) and human (18-22) compared to sets of control genes ( $p$ $<0.005$ for all, z-test). For all panels except (C), error bars show \pm one standard deviation across 20 sets of control genes. (B) GIMs are enriched in genes with low tolerance for loss-of-function mutations in humans $(24)\left(p=1.2 \times 10^{-7}\right.$, z-test). (C) Gamete-level TRD genes in mouse (25) include four genes with extremely high genoinformativity scores. (D) GIMs are enriched for genes with evidence for bovine paternal-specific TRD (26) ( $p=5.3 \times 10^{-22}$, z-test). (E) GIMs are enriched for testis-specific expression in mice (36) but not human (37). (F) GIMs represent a higher number of paralog families than non-GIMs in mice and humans $(38)(p=$ $1.4 \times 10^{-66}, p=9.6 \times 10^{-13}$, z-test). (G) GIMs are enriched in testis-specific exons in mice (39) and testis-specific isoforms in humans (37). (H) Mouse GIMs that are functional candidates are enriched for specific late translation (28) $\left(p=1, p=3.0 \times 10^{-4}, p=2.6 \times 10^{-43}, p=8.4 \times 10^{-11}\right.$, left to right, Fisher's exact test). The GIMs are taken from the blue bars in (A) to (D) and are compared to GIMs not in that category. 